Original Research Paper

\title{
cDNA Cloning and Characterization of the House Dust Mite Allergen Sui p 2
}

\author{
${ }^{1,2}$ Joshua Evans M. Bajao and ${ }^{1,3,4}$ John Donnie A. Ramos \\ ${ }^{I}$ The Graduate School, University of Santo Tomas, España Blvd., Manila, Philippines \\ ${ }^{2}$ Senior High School, University of Santo Tomas, España Blvd., Manila, Philippines \\ ${ }^{3}$ Department of Biological Science, College of Science, University of Santo Tomas, España Blvd., Manila, Philippines \\ ${ }^{4}$ Research Center for the Natural and Applied Sciences, University of Santo Tomas, España Blvd., Manila, Philippines
}

\author{
Article history \\ Received: 30-10-2019 \\ Revised: 22-01-2020 \\ Accepted: 13-05-2020 \\ Corresponding Authors: \\ Joshua Evans M. Bajao \\ The Graduate School, \\ University of Santo Tomas, \\ España Blvd., Manila, \\ Philippines \\ Email:jmbajao@ust.edu.ph
}

\begin{abstract}
Suidasia pontifica (Sp) is a House Dust Mite (HDM) species found in tropical urban residential areas which causes sensitization of up to $75 \%$ of allergic patients. Cloning of allergens from $\mathrm{Sp}$ is a significant initial step in understanding the clinical significance of this HDM species. The study describes the cloning and in silico characterization of the group 2 allergen gene from $\mathrm{Sp}$ (Sui $\mathrm{p}$ 2) with significant homology to Myeloid Differentiation 2 (MD-2) lipid-binding (ML domain) protein allergens. Sui p 2 gene was amplified by Reverse Transcription - PCR using gene-specific primers designed from known HDM group 2 allergens. The amplicons were characterized using NCBI BLAST, ExPASy, MEGA7 and Chimera. Sui p 2 cDNA gene exhibits percent identity to Tyrophagus putrescentiae (65.84\%), Aleuroglyphus ovatus (65.69\%) and Blomia tropicalis (62.98\%). Sui p 2 gene contains 405 bp open reading frame with untranslated regions on its 5' and 3' end. Furthermore, structure-based percent identity of Sui p 2 to the group 2 allergen of D. farinae and D. pteronyssinus is $47.62 \%$ and $46.83 \%$, respectively. Predicted protein structure reveals a putative immunoglobulin fold consisting of preserved cysteine residues, hydrophobic core and two antiparallel $\beta$-sheets containing several TLR4, LPS and IgE binding sites. The results suggest that Sui p 2 may play an important role in HDM induced allergy and may be involved in the induction of the innate immune system. Characterization of Sui p 2 is important to evaluate properly its use for allergy component resolved diagnosis and allergen-specific immunotherapy.
\end{abstract}

Keywords: Allergen, House Dust Mite, Innate Immunity, T-cell Epitope, Toll-Like Receptors

\section{Introduction}

Allergy is a hypersensitivity reaction commonly characterized by excessive production of $\operatorname{IgE}$ that is specific to environmental proteins called allergens (Peters et al., 2013). Epidemiological studies show that around ten to fifty percent $(10-50 \%)$ of the world's population suffers from at least one of the diverse forms of allergic diseases such as atopic dermatitis, allergic rhinitis and asthma (Bergmann et al., 2016; Pawankar et al., 2012). Predisposition to allergy is highly dependent to the genetic background of an individual (Wang et al., 2012); however, sensitization of atopic patients are triggered by common environmental proteins called allergens (Lau et al., 2014; Hanski et al., 2012). The most common sources of indoor allergens and exhibiting the highest rate of sensitization are the House Dust Mites (HDMs) (Farrokhi et al., 2015).

HDMs are astigmatid arachnids that feed on skin dander and other organic detritus found in beddings, carpets, stuffed toys, fabrics and other parts of the house (Ciftci et al., 2006). Hypersensitivity to its allergens are one of the most common cause of allergic reactions in the world. Up to $90 \%$ of allergic patients worldwide are sensitized to HDM allergens (Yong et al., 2013). However, the allergen profiles of different localities may differ due to the difference of diversity and number of HDM species per region (Ramos et al., 2007). Hence, it is important to study HDMs in a particular area to properly elucidate its clinical importance. One of these locally-isolated allergenic HDM species that has not yet been thoroughly studied is the Suidasia pontifica $(\mathrm{Sp})$. 
Also known as Suidasia medanensis, $\mathrm{Sp}$ is a relatively ubiquitous HDM present in approximately $80 \%$ of the homes in the tropics (Mariana et al., 2000). $\mathrm{Sp}$ is capable of causing sensitization of allergic patients (Yap et al., 2014). In extreme cases, Sp allergens can elicit oral mite anaphylaxis especially when allergic individuals consumed Sp-infested food items such as rice flours and cereals (Mariana et al., 2010; Sanchez-Borges et al., 2013). Despite having a high rate of sensitization, the allergenicity of Sp has not yet been properly evaluated. Thus, specific allergens from $\mathrm{Sp}$ must be isolated and characterized to assess its clinical importance properly.

Designated as the group 2 allergen in HDMs, the myeloid differentiation-2-like (ML) lipid-binding domain is a family of proteins that is structurally similar to the myeloid differentiation-2 (MD-2) protein (Dall'Antonia et al., 2014). The MD-2 is responsible for the initiation of the mammalian innate immune system against bacterial Lipopolysaccharides (LPS), through Toll-Like Receptor-4 (TLR4) binding (Ryu et al., 2017). As an allergen, the ML lipid-binding domain protein is one of the three serodominant HDM allergens and is one of the most common allergens in the environment (Thomas, 2015; Hernández-Cadena et al., 2015). In terms of its IgE-binding activity, the HDM group 2 allergen is the most potent HDM allergen, capable of binding to up to $100 \%$ of the IgEs present in the body of an atopic patient (Jeong et al., 2012). Furthermore, the said allergen exhibits the highest rate of sensitization, together with the HDM group 1 allergen, among the other HDM allergens (Jeong et al., 2015).

We described in this paper, the cloning and in silico characterization of an ML lipid-binding domainhomolog allergen from $\mathrm{Sp}$ (Sui p 2). The resulting sequence was characterized through computational biology and in comparison with other known HDM group 2 allergens and the mammalian MD-2.

\section{Methods}

\section{Suidasia Pontifica Mite Cultures and mRNA Isolation}

Monocultures of Suidaisa pontifica ( $\mathrm{Sp}$ ) was obtained from UST-RCNAS. The monocultures were placed in a BD Falcon ${ }^{\circledR} 50 \mathrm{~mL}$ tissue culture flasks (BD Biosciences, San Jose, CA, USA) and were fed with powdered and sterilized TetraMin® fish flakes (Tetra Werke, Germany) every 2 weeks. The humidity was loosely controlled using a beaker filled with water and the cultures were maintained with temperatures ranging from $24-30^{\circ} \mathrm{C}$. The mites were harvested using a modified Tullgren apparatus and were stored at $-20^{\circ} \mathrm{C}$ until analysis. Total RNA was extracted from isolated $\mathrm{Sp}$ cultured mites using Wizard ${ }^{\circledR}$ SV Total RNA Isolation Kit (Promega, Madison, WI,
USA) according to manufacturer's protocol. The isolated total mRNA was stored at $-70^{\circ} \mathrm{C}$.

\section{Reverse Transcription-Polymerase Chain Reaction (RT-PCR)}

The complementary DNA (cDNA) was synthesized from the total RNA using Tetro cDNA Synthesis Kit (Tauton, MA, USA). Six $\mu \mathrm{L}$ of the total RNA were mixed into a solution containing $1 \mu \mathrm{L}$ Oligo(dT) (Hernández-Cadena et al., 2015) primer; $1 \mu \mathrm{L} 10 \mathrm{mM}$ dNTP mix; $4 \mu \mathrm{L}$ RT buffer; $1 \mu \mathrm{L}$ RiboSafe RNase inhibitor; $1 \mu \mathrm{L}$ Tetro Reverse Transcriptase; and, $6 \mu \mathrm{L}$ DEPC-treated water. cDNA synthesis was performed by incubating it at $45^{\circ} \mathrm{C}$ for $30 \mathrm{~min}$, the reaction was terminated by incubating the reaction mixture at $85^{\circ} \mathrm{C}$ for five minutes. The cDNA was immediately stored at $20.0^{\circ} \mathrm{C}$ until use. The full length Sui p 2 gene was isolated using Polymerase Chain Reaction (PCR) from the synthesized cDNA. Five primers, consisting of three forward (MLF1.1: TGGTGAAGTTCATCATCTTGGC; MLF1.2: TGATGAAGTTCACATCTTGGCT; and MLF2: CGATAAGGAGTGGACGGGAA) and two reverse (MLR1: AGTTTAGTTCTGGACCTCACTGT; and MLR2: TACCATCTCTTCGCGTCGTG), was used to create three primer pairs. The primers were designed based on consensus sequences from other known HDM group 2 allergen genes using OligoAnalyzer 3.1 (IDT, Coralville, Iowa, USA).

The amplicons were verified using a $1 \%$ Agarose Gel (Vivantis, Oceanside, California, USA). Ethidium bromide (Sigma-Aldrich, St. Louis, Missouri, USA) was used to detect the presence of the amplicons in the said agarose gel. For Sequencing, the components in the PCR was increased five times, having a final volume of 50 $\mu \mathrm{L}$. Together with a $100 \mu \mathrm{L}$ both forward and reverse primers, the amplicons were sent and sequenced in Macrogen (Geumcheon-gu, Seoul, Korea).

\section{In silico Analysis of Sui p 2 Gene}

The identity of the amplicons were verified using NCBI BLAST (Altschul et al., 1990). The Open Reading Frames (ORF) of all the sequences as well as the deduced amino acid sequence were determined ExPASy's translate tool (Gasteiger et al., 2003). The ORFs of the HDM group 2 allergen nucleotide sequence, as well as that of the mammalian MD-2 nucleotide sequences, were aligned using Clustal OMEGA (Sievers et al., 2011) and a phylogenetic tree was constructed and viewed using Molecular Evolutionary Genetic Analysis ver.7 (MEGA7) (Kumar et al., 2016) and FigTree (http://tree.bio.ed.ac.uk/software/figtree/), respectively. The appropriate model used for phylogenetic tree construction was determined using the model selection tool of MEGA7. The model used for the phylogenetic tree is the Kimura 2-parameter model. 
Table 1: Tertiary structure prediction and assessment of Sui $\mathrm{p} 2$ models

\begin{tabular}{|c|c|c|c|c|c|c|c|c|}
\hline \multirow[b]{4}{*}{ Clone } & \multirow{4}{*}{$\begin{array}{l}\text { 3D protein } \\
\text { modelling tools }\end{array}$} & \multicolumn{7}{|c|}{ Protein quality assessment tool } \\
\hline & & \multicolumn{2}{|c|}{ QMEAN Server } & \multicolumn{2}{|l|}{ ProQ } & \multirow{3}{*}{$\begin{array}{l}\text { ProSa-Web } \\
\text { Overall } \\
\text { model quality }\end{array}$} & \\
\hline & & \multicolumn{2}{|l|}{ QMEAN } & \multirow[b]{2}{*}{ LG score } & \multirow[b]{2}{*}{ Max score } & & \multicolumn{2}{|l|}{ Saves } \\
\hline & & score & Z score & & & & $\operatorname{ERRAT}(\%)$ & Verify3D (\%) \\
\hline \multirow[t]{4}{*}{ Suip2.01 } & Swiss-Model & 0.656 & 0.90 & 1.892 & 0.259 & -4.58 & 82.759 & 96.77 \\
\hline & Phyre $^{2}$ & 0.629 & 1.21 & 2.417 & 0.282 & -4.59 & 82.906 & 94.44 \\
\hline & CPHmodels 3.2 & 0.609 & 1.41 & 2.919 & 0.382 & -4.66 & 70.690 & 100 \\
\hline & Raptorx & 0.735 & -0.05 & 1.767 & 0.272 & -4.52 & 94.068 & 100 \\
\hline \multirow[t]{4}{*}{ Suip2.02 } & Swiss-Model & 0.650 & 0.96 & 2.826 & 0.329 & -4.45 & 78.488 & 98.39 \\
\hline & Phyre $^{2}$ & 0.626 & 1.24 & 2.960 & 0.348 & -4.54 & 88.136 & 100 \\
\hline & CPHmodels 3.2 & 0.604 & 1.46 & 2.717 & 0.372 & -4.48 & 70.690 & 100 \\
\hline & Raptorx & 0.711 & 0.32 & 2.499 & 0.322 & -4.94 & 81.356 & 100 \\
\hline \multirow{4}{*}{ Suip2.03 } & Swiss-Model & 0.605 & 1.47 & 2.288 & 0.319 & -4.42 & 65.217 & 100 \\
\hline & Phyre $^{2}$ & 0.596 & 1.57 & 2.443 & 0.302 & -4.69 & 74.576 & 92.86 \\
\hline & CPHmodels 3.2 & 0.591 & 1.63 & 3.104 & 0.398 & -4.62 & 84.348 & 100 \\
\hline & Raptorx & 0.784 & 0.48 & 1.724 & 0.246 & -4.18 & 66.129 & 91.04 \\
\hline
\end{tabular}

The presence of a signal peptide to the deduced amino acid sequences were determined using SignalP 4.1 server (Petersen et al., 2011). The protein parameters of the deduced Sui p 2 sequences revealed by ExPASy's ProtParam tool (Gasteiger et al., 2005). The tertiary structure of the deduced Sui p 2, without its signal peptide, was predicted using four prediction servers, namely: ExPASy's Swiss-Model workspace (Arnold et al., 2006); Protein Homology/analogY Recognition Engine V 2.0 (Phyre ${ }^{2}$ ) (Kelley et al., 2015); CPHmodels 3.2 Server (Nielsen et al., 2010); and, RaptorX (Källberg et al., 2012). The deduced model was compared to the crystallographic models of $D$. pteronyssinus (1ktj) group 2 allergens. The protein quality of the predicted tertiary structures of Sui p 2 was predicted using ExPASy's QMEAN server (Benkert et al., 2008), SBC's Protein Quality (ProQ) (Wallner and Elofsson, 2003), Protein Structure Analysis (ProSA-Web) (Wiederstein and Sippl, 2007) and UCLA Structure Analysis and Verification Server (SAVES) (Pontius et al., 1996). The deduced model who has the greatest similarity to the said HDM group 2 allergens, while having the best protein quality (Table 1), was chosen for the in silico characterization of Sui $\mathrm{p} 2$. The predicted tertiary structures were viewed and analyzed using Chimera (Pettersen et al., 2004) and are compared to other HDM group 2 allergens obtained in RCSB PDB.

The chosen model was compared to other PDB models of the ML family. Four models were considered: the 129 aa long Der $f 2$ (PDB ID: 1xwv); the 129 aa Der p 2 (PDB ID: 1ktj); the 160 aa human MD-2 (hMD-2), PDB ID: 2e56 chain C; and, the 157 aa mouse MD-2 (mMD-2), PDB ID: $5 \mathrm{hg}^{3}$ chain C. All models were acquired using $\mathrm{X}$-ray diffraction analyses. The models were aligned, through chimera, based on the comparison of its structural information through superimposition. The resulting alignment was viewed in ESPript3.0 (Robert and
Gouet, 2014). The percent identities of Suip2.01 to other ML family are: Der f 2 at $47.62 \%$; Der p 2 at $46.83 \%$; hMD-2 at $15.08 \%$; and, mMD-2 at $12.70 \%$.

\section{Results}

\section{Sui $p 2$ Exhibits Homology to HDM Group 2 Allergens}

RT-PCR of the Sui $\mathrm{p} 2$ cDNA gene yielded three amplicons of approximately $430 \mathrm{bp}$ products referred to as Suip2.01 and Suip2.02 using MLF1.1 and MLR1 primer pair; and Suip2.03 using MLF1.2-MLR1 primer pair. No PCR products were amplified using the MLF2MLR2 primer pair. After sequencing, the sequences were submitted to NCBI with accession numbers KY449406.1 (Suip2.01), KY449407.1(Suip2.02) and KY449408.1 (Suip2.03). All the clones contained a 405 bp long ORF translated into a 134 bp long aa sequence (Fig. 1). All of the clones are almost identical with each other with minor sequence variations on the last ten nucleotides.

The sequences of the amplicons were compared to 25 known HDM group 2 allergens from nine different HDM species. Four samples of mammalian MD-2 were used as the outgroup. However, some of the nucleotide sequences obtained from NCBI contain coding regions only. Thus, to minimize discrepancies, only the ORFs were used for sequence alignment. Among the HDM species, $T$. putrescentiae has the highest sequence homology to Sui p 2 while $D$. farinae has the lowest, having a $65.84 \%$ and $50.26 \%$ homology, respectively. Using the ORFs of the allergen as well as that of the outgroups, a phylogenetic tree was constructed (Fig. 2).

A 134 aa sequence was deduced using the ORF of the Sui p 2 gene. According to ExPASy's ProtParam (Gasteiger et al., 2005), the deduced Sui p 2 is a 14.3 $\mathrm{kDa}$ protein that is relatively stable and neutral. 
Sequence comparison of Sui $\mathrm{p} 2$ to other HDM group 2 allergens, as well as to mammalian MD-2 showed that Aleuroglyphus ovatus group 2 allergen has the highest homology, at $63.43 \%$, to Sp. However, there are only $16.67 \%$ and $17.42 \%$ sequence homology of Sui $\mathrm{p} 2$ to mMD-2 and hMD-2, respectively.

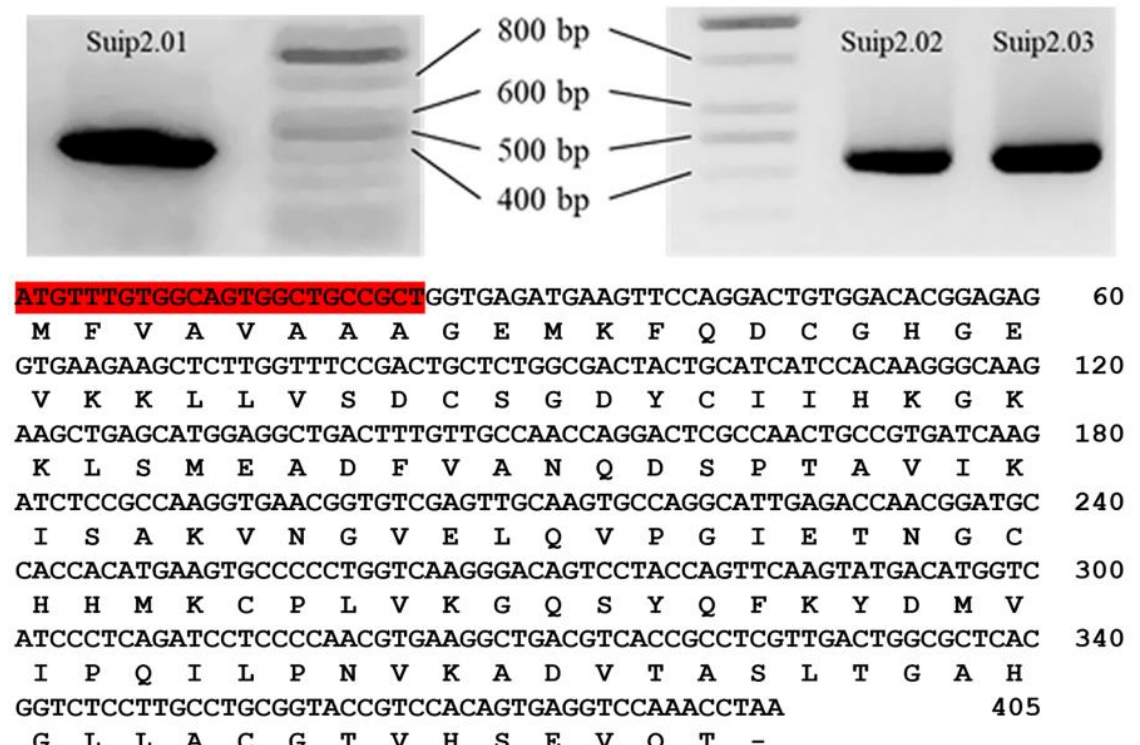

Fig. 1: Agarose gel profiles of the RT-PCR amplified group 2 allergen from Suidasia pontifica Sui p 2 (above) and its nucleotide and deduced amino acid sequences (below). Except at the last ten nucleotides (bold), all of the sequences of the clones are identical to each other. The nucleotide/aa sequence of Suip2.02 and Suip2.03 is CAAAAAATAA/KK- and AGAAAAATAA/EK-, respectively. The signal peptide (red) cleavage site was predicted to be located between Ala8 and Gly9

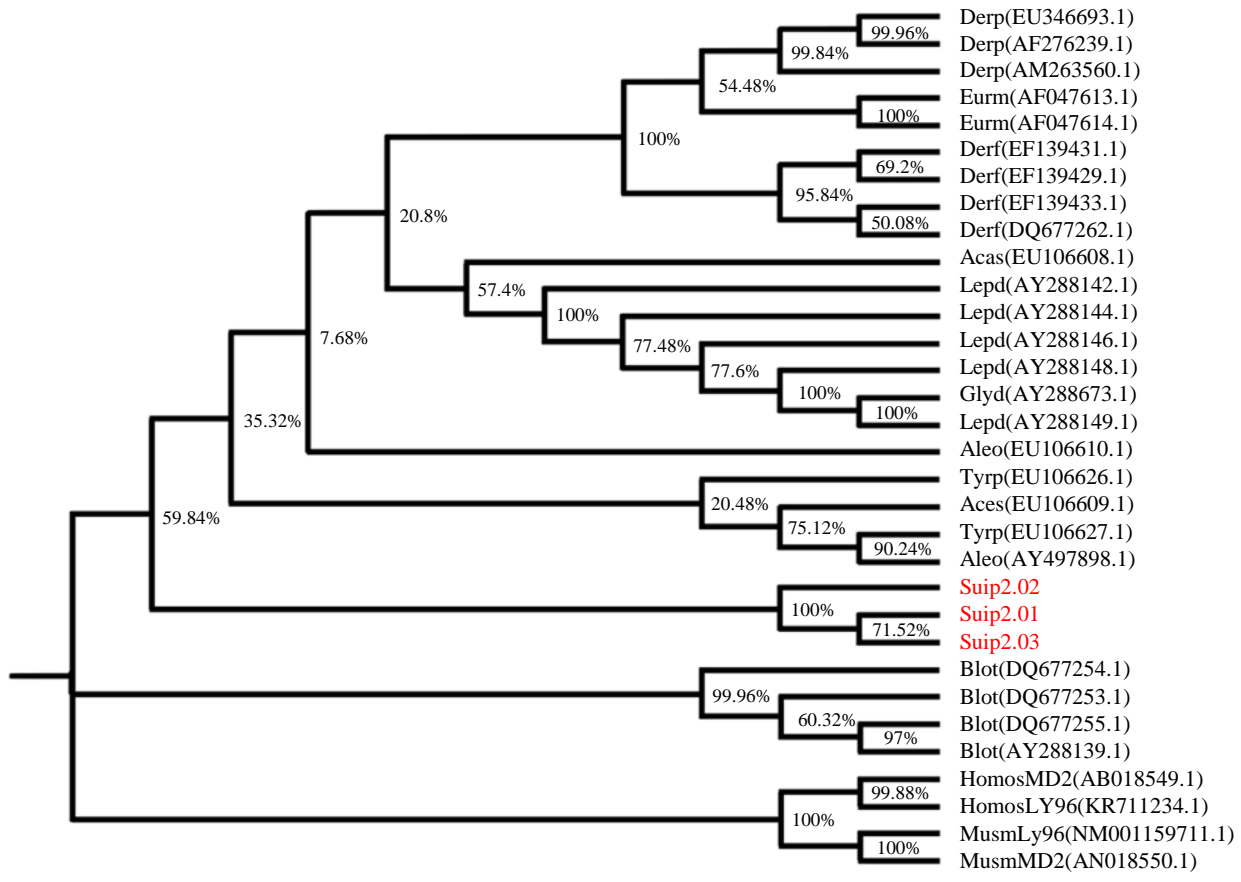

Fig. 2: The phylogenetic tree of HDM group 2 allergen with four mammalian MD-2 genes. The bootstrap consensus tree inferred from 2500 replicates is taken to represent the evolutionary history of HDM group 2 allergen. Sui p 2 clones (red) have formed a single group within the deduced phylogenetic tree 
The deduced amino acid sequences from the 3 Sui $\mathrm{p} 2$ clones all contain eight aa long signal peptides, as analyzed by SignalP4.1 software (Petersen et al., 2011). The signal peptide cleavage site is located between Ala8 and Gly9 (Fig. 1). The presence of the signal peptide is consistent with the amino acid sequences of other group 2 allergens (Cui et al., 2010; Horáčková et al., 2010) thus indicating that the Sui p 2 cDNA genes described in this study are the full-length sequences of the Sp group 2 allergen. Being an extracellular protein, the signal peptide present in Sui p 2 may play a major role towards the transport of the protein to the outside of the cell. The deduced 134 amino acid long sequence of Sui p 2 has a predicted Isoelectric point (pI) of 6.57; extinction coefficient of 0.339 ; instability index of 24.89; aliphatic index of 87.99; and grand average of hydropathicity (GRAVY) of 0.065 .

\section{In silico Modelling of Sui $p 2$ and its Basic Protein Parameters}

Using the deduced amino acid sequence, twelve tertiary structure models were produced using four protein model prediction servers, including SwissModel, Phyre ${ }^{2}$, CPHmodels-3.2 server and RaptorX (Fig. 3). All of the three models created by Phyre ${ }^{2}$ but excluding the Suip2.02R due to the presence of only two disulfide bridges instead of three as observed in the other sequences. Suip2.01S and Suip2.02S were removed due to the absence of a single $\alpha$-helix in its chain while Suip2.03S and Suip2.03C were eliminated because of the absence of a parallel $\beta$-sheet. Lastly, models Suip2.1C and Suip2.02C are also excluded because there are two closed cavities inside its surface instead of a single cavity with a mouth. Among the twelve models, only Suip2.01R and Suip2.03R showed similarity to the crystallographic image of Der p 2 (1ktj). Model
Suip2.01R was chosen as the standard model because it has the best protein quality according to various protein prediction servers.

The 126 aa long model has a molecular weight of 13528.6 Da with a theoretical isoelectric point $(\mathrm{pI})$ of $\mathrm{pH}$ 6.63 while its GRAVY is -0.092 , making it slightly hydrophilic yet slightly acidic. It also has an instability index of 25.84, making it stable when purified and stored (Gasteiger et al., 2005). The aliphatic index of Suip2.01 is 85.79 , thus a potential thermostable protein.
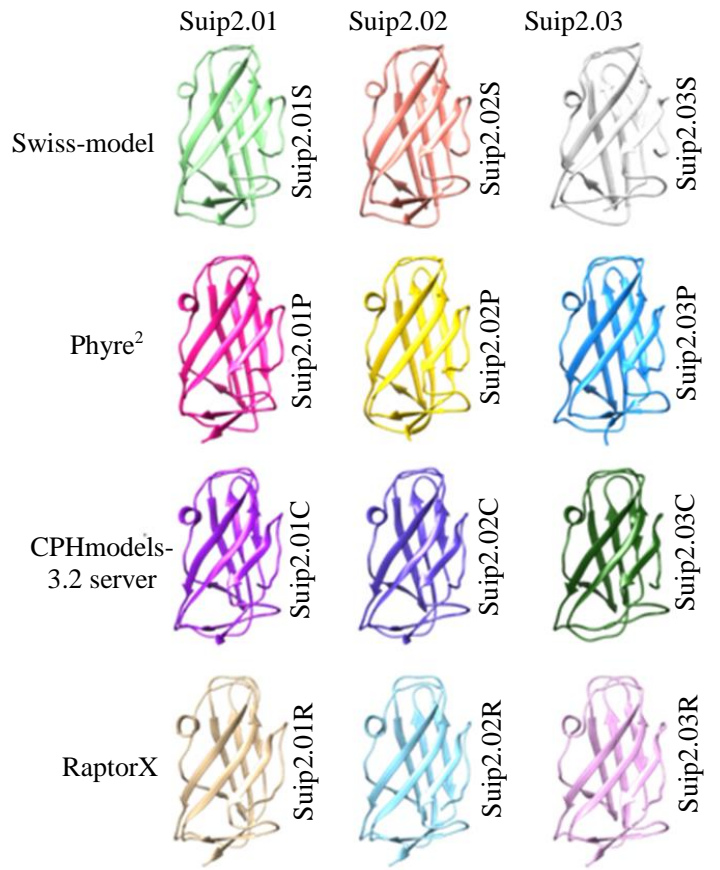

Fig. 3: Tertiary structure models of Sui p 2 from four different prediction servers using all of the clones

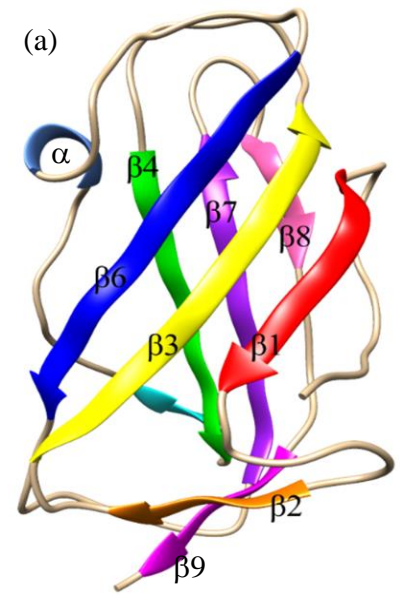

(b)

$\begin{array}{lc}\text { GEMKFQDCGHGEVKKLLVSDCSGDYCIIH } & 30 \\ \text { GKKLSMEADFVANQDSPTAVIKISAKVNGV } & 60 \\ \text { ELQVPGIETNGCHHMKCPLVKGQSYQFKYD } & 90 \\ \text { MVIPQILPNVKADVTASLTGAHGLLACGTV } & 120 \\ \text { HSEVQT } & 126\end{array}$

(c)

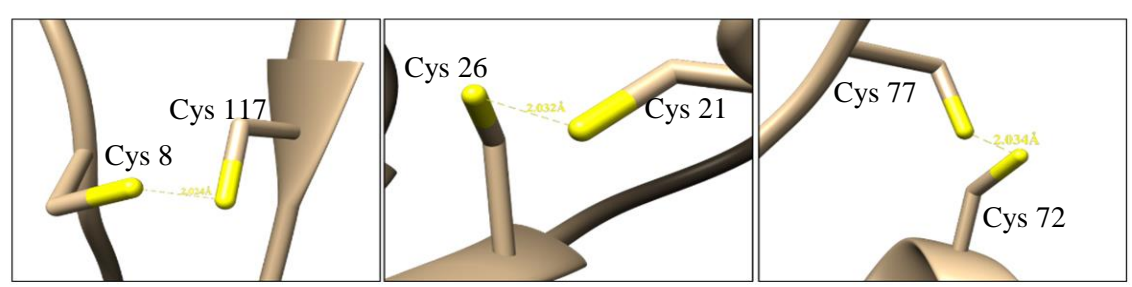

Fig. 4: The tertiary structure model of Suip2.01 (a) alongside its deduced amino acid sequence (b). The color on both the nucleotide and the amino acid sequence is parallel to that of the structure. The entire structure is stabilized by three disulfide pairs (c) 


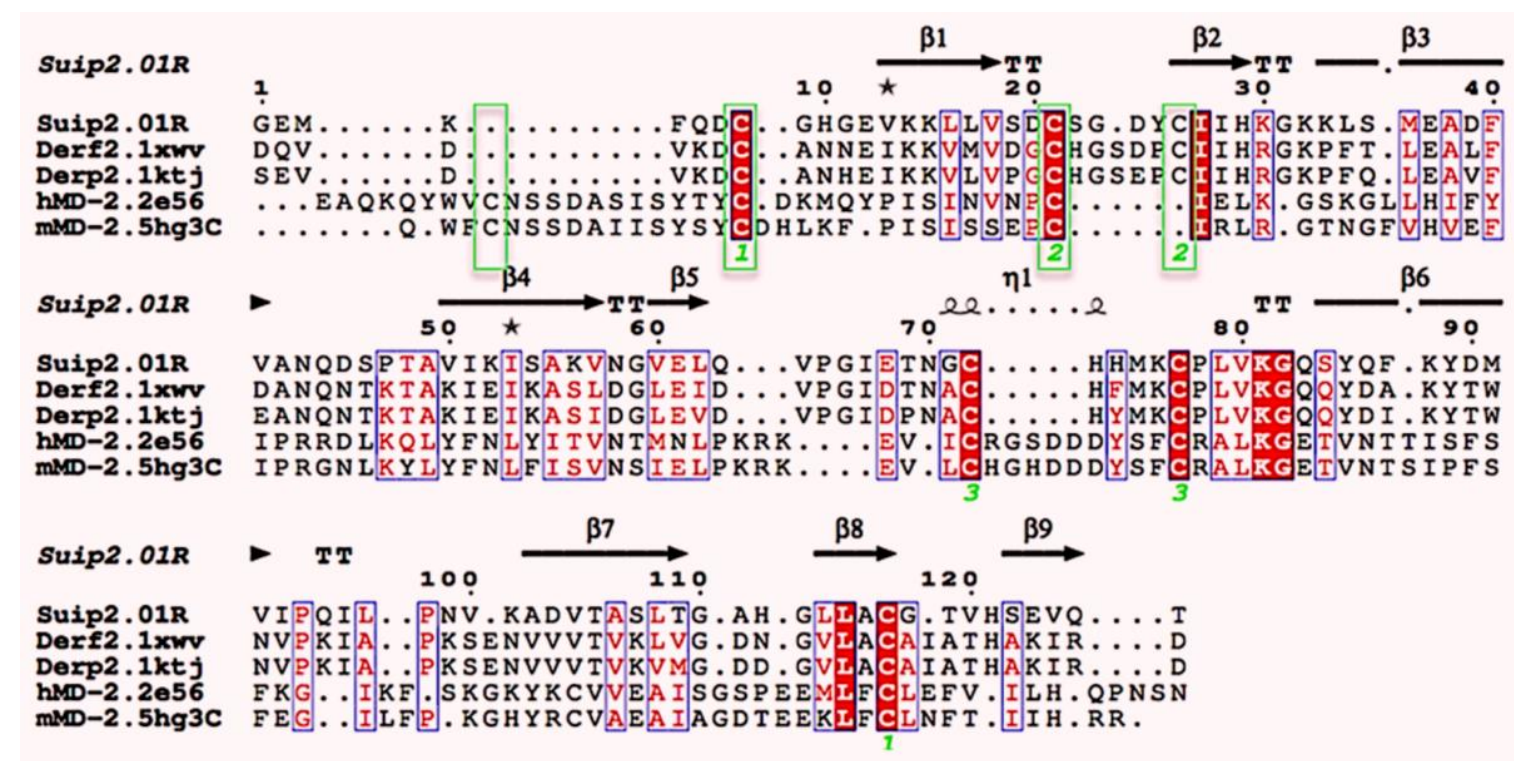

(a)

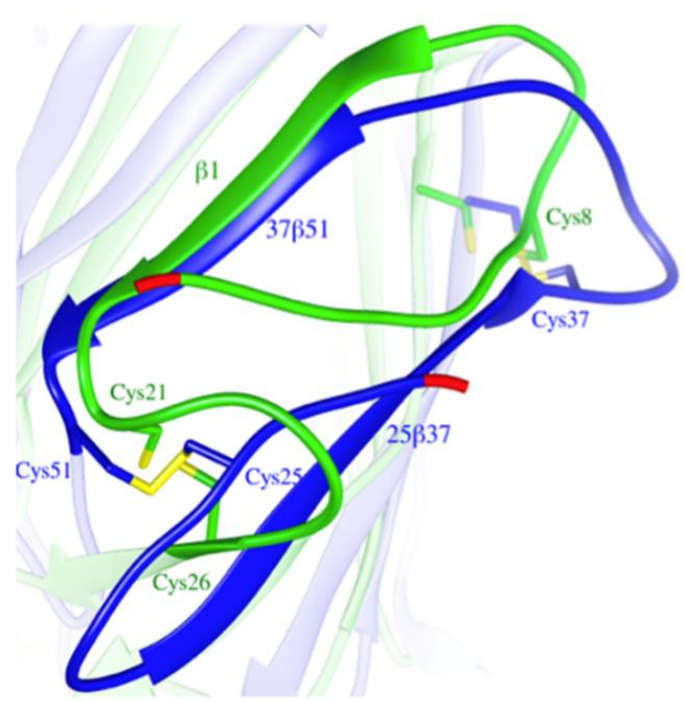

(b)

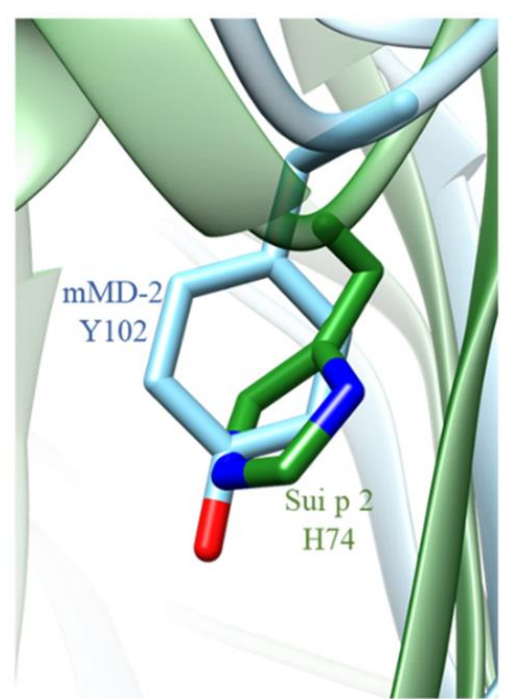

(c)

Fig. 5: Sui p 2 sequence alignment to other proteins in the ML lipid-binding domain family (a). Although there are differences between the di-sulfide bridges of mammalian MD-2 and HDM group 2 allergens, the cysteine pairing is conserved through all of the models (pairs in green box). The Sui p 2 (green) and the mMD-2 superimposed models showing their first disulfide bridge (b). However, instead of the Tyr102 (cyan) of mMD-2, Sui p 2 has His74 at the same location as the Tyr102 (c). Tyr102 anchors the LPS through the hydrogen bonding of the OH group (red) to the R3' acyl chain of Lipid IVa to mMD-2. Hydrogen bonding of Suip2.01 can be due to the nitrogen (cyan) part of the imidazole ring of His74

Around $43.65 \%$ and $2.38 \%$ of the amino acid of Sui $p$ 2 formed nine $\beta$-strands $(\beta 1-\beta 9)$ and one $\alpha$-helix, respectively (Fig. 4). Of the nine predicted $\beta$-strands, seven are involved in the formation of two antiparallel $\beta$ sheets. However, two of the $\beta$-strands, $\beta 1$ and $\beta 9$, form a parallel configuration to each other. Because of the configuration of the entire model, Sui p 2 appear to follow an S-type configuration (Bork et al., 1994). The six cysteine residues of Sui p 2 are predicted to be oxidized, forming a disulfide bridge with another Cysteine residue. The disulfide pairs are Cys8-Cys117, Cys21-Cys26 and Cys72-Cys77 (Fig. 5C).

\section{Sui 2 as an ML Lipid-Binding Domain - Homolog}

In order to elicit the innate immune system, there are certain features of the MD-2 that are needed for TLR4MD-2-LPS binding and one of it is its hydrophobic cavity. There are 37 amino acids that contribute to the 
surface of the internal cavity of the Suip2.01 model, 33 of which are hydrophobic in nature. Per-model clipping of the rendered surface of the protein, based on the KyteDoolitle hydrophobicity scale, showed that the majority of the surface are hydrophobic. Also, two openings of the cavity has been predicted. One of the openings is at the bottom of the $\alpha$-helix while the other is at the opposite side of the protein.

The Sui p 2 model was compared to the PDB models of Der f 2, Der p 2, hMD-2 and mMD-2. The percent homology of Sui p 2 to other ML family are: Der f 2 at 47.62\%; Der p 2 at $46.83 \%$; hMD-2 at $15.08 \%$; and, mMD2 at $12.70 \%$. All of the disulfide bridges have been conserved in all ML family. However, the hMD-2 and the mMD-2 have seven cysteine residues that are identical to each other in terms of location. Among the seven cysteine residues, only six are oxidized and form three disulfide bridges while Cys 133 of both proteins remains unpaired. In terms of the location of the di-sulfide bridges, only the first and the third pairs are structurally similar to each other (Fig. $5 \mathrm{~A})$. Because of the difference in the bonding pair of the disulfide bridges, there is a shift in $\beta$-strand composition of the two $\beta$-sheets (Fig. 5B). Despite their stark differences, the S-type configuration of the immunoglobulin fold has been conserved in all of the proteins.

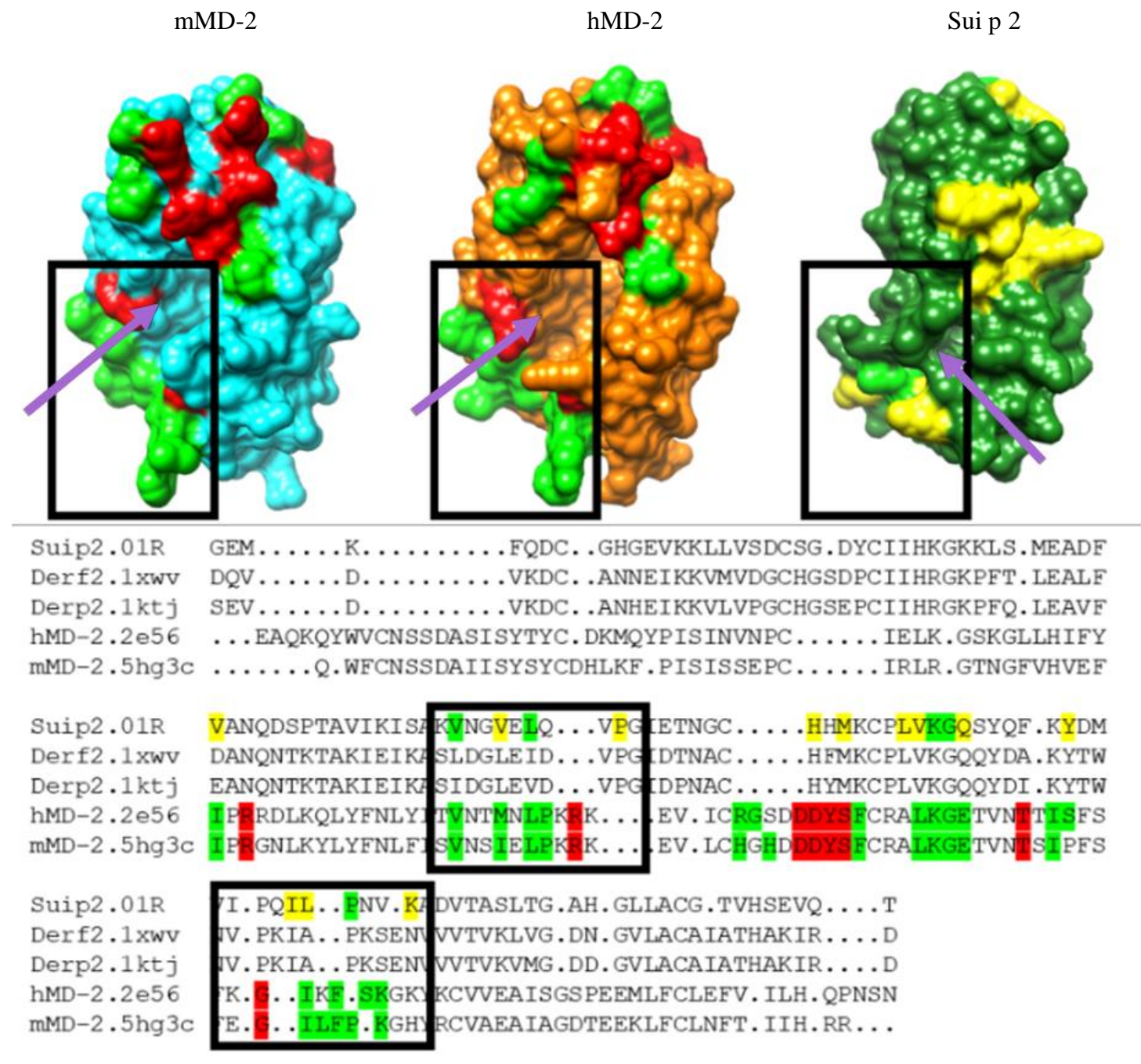

Fig. 6: TLR4 and binding site of mMD-2, hMD-2 and Suip2.01. The TLR4 binding site of both MD-2 proteins that are present and absent in Suip2.01 are colored in light green and red, respectively. The TLR4 binding sites that are strictly conserved in Suip2.01 are colored in light green while the presumed, due to similar properties, TLR4 binding sites are colored in yellow. The TLR4'dimerization binding site of mMD-2, hMD-2 and Sui $\mathrm{p} 2$ is enclosed in a square. One of the openings of the hydrophobic cavity (purple arrow) is near the TLR4' dimerization binding site. The sequence alignment of all included MD-2 and HDM group 2 allergen showing the TLR4 binding sites of Suip2.01, hMD-2 and mMD-2. The TLR4' dimerization binding sites on the sequence are enclosed in a box (b). The highlighted portions on the sequence are parallel to the proteins above 
Comparison of Sui $\mathrm{p} 2$ to both mMD-2 and hMD-2 revealed that there are possible amino acid residues that are capable of binding to TLR4 (Fig. 6). Based on the nature of the binding, two distinct binding sites were assessed: The TLR4 binding site for the primary TLR4; and, the TLR4' binding site for the TLR4 that is responsible for dimerization. There are 17 sites present in hMD-2 and mMD-2 (Ohto et al., 2012; Walsh et al., 2008) that are responsible to TLR4 binding; ten sites might be present in Sui p 2 and only two are strictly conserved in terms of its sequence. The conserved amino acids of Sui p 2 are Lys81 and Gly82, which are homologous to Lys109 and Gly110 of both mMD-2 and hMD-2, respectively. Some of the amino acids were still considered as possible binding sites due to its similarity to known binding sites. Furthermore, there are 11 secondary TLR4 (TLR4') binding sites present on both MD-2 (Ohto et al., 2012; Walsh et al., 2008); eight of it may be present in Sui $\mathrm{p}$ 2 and two are strictly conserved in Sui $\mathrm{p} \mathrm{2.} \mathrm{The}$ conserved amino acids of Sui p 2 are Val57 and Leu62, which are homologous to Val82 and Leu87 of both hMD-2 and hMD-2. Also, the said binding site Val57 (Sui p 2) can be found on Der f 2 and Der p 2 but as leucine and isoleucine, respectively. Like Val57, the binding site Leu62 (Sui p 2) can be found on Der f 2 and Der p 2 as Ile63 and Val63, respectively. Similar to the TLR4 binding sites, some of the amino acids were still deemed as possible binding sites for TLR4'.

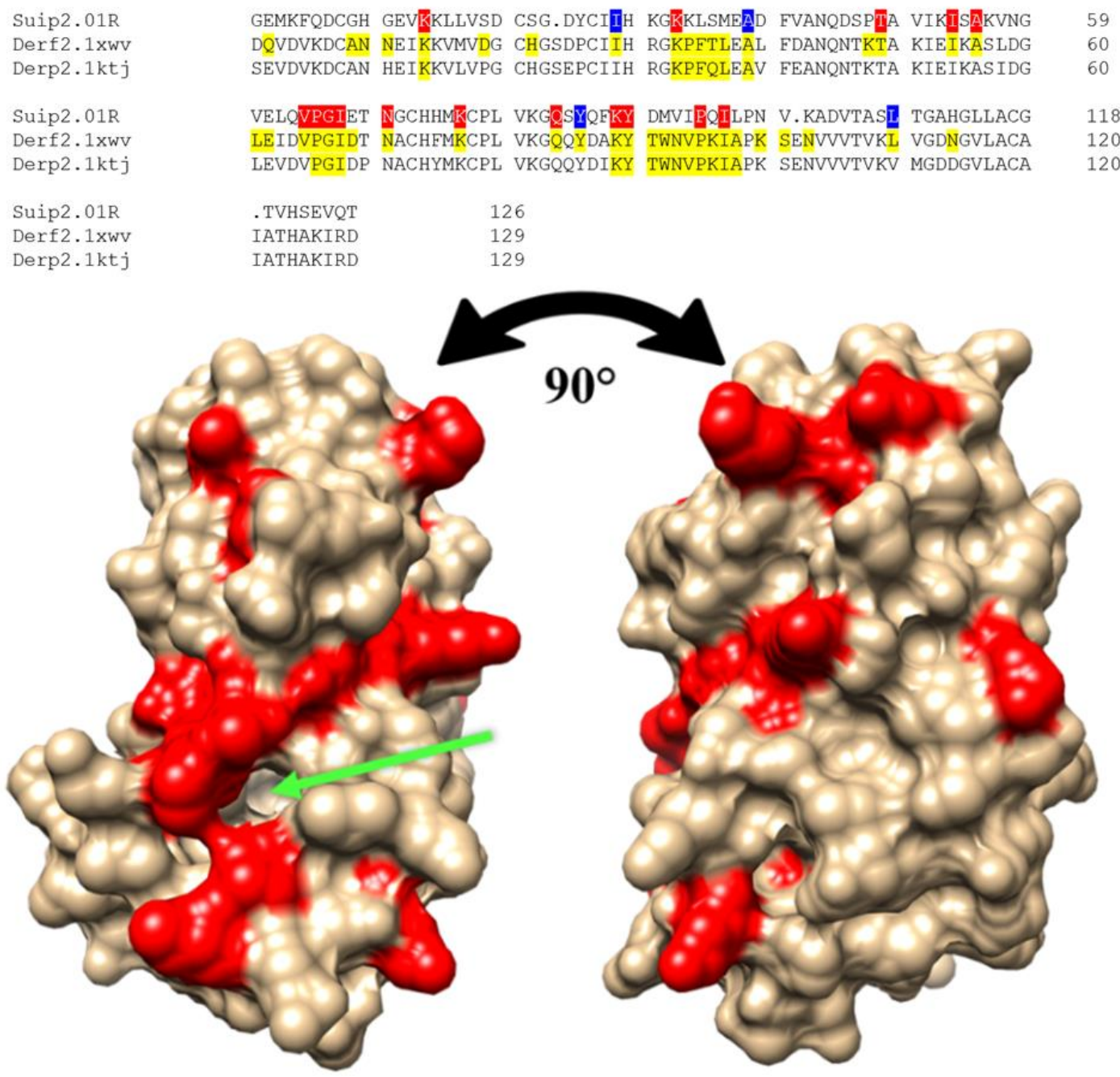

Fig. 7: Putative IgE-binding epitope of Suip2.01. The aligned sequence of Suip2.01 to Der f 2 and Der p 2 (above). The epitopeforming amino acids of Der f 2 and Der $\mathrm{p} 2$ and are highlighted in yellow while its amino acid counter parts in Suip2.01 are highlighted in red, which is reflected to the surface rendering of Sui $\mathrm{p} 2$ (below). However, four conserved amino acids were excluded due to the lack of accessibility of the side chains to the surface of the protein (blue). The majority of the presumed epitope is localized near the opening of the hydrophobic cavity (green arrow) 


\section{Sui $p 2$ has a Putative Epitope-Forming Amino Acid Residues}

The possible IgE-binding epitopes of Suip2.01 was deduced by comparing it to the aa sequences of Der $f$ and Der $\mathrm{p} 2$. Overall, there are 44 epitope-forming amino acids on Der f 2 and Der p 2 (Szalai et al., 2007), 25 of which are unique to Der f 2 (Ichikawa et al., 2005). Out of the said epitope-forming amino acids, 20 amino acids are conserved in Suip2.01 and are considered to be a putative epitope-forming amino acid (Fig. 7). However, out of the twenty conserved amino acids that are considered, Ile28, Ala38, Tyr85 and Leu108 excluded. Based on the rendered surface of Sui $\mathrm{p} 2$, these amino acids are accessible only at the hydrophobic cavity. Hence, they are not accessible for IgE binding.

\section{Discussion}

Sui p 2 gene has a relatively low sequence homology to ML from other HDM, having only a $65.84 \%$ sequence homology to Tyr p 2 gene. This low sequence homology was further supported when Sui p 2 gene was compared to mammalian MD-2, having only a $39.82 \%$ and $40.41 \%$ sequence homology to mMD-2 and hMD-2 gene, respectively. Due to the low sequence homology of the Sui p 2 gene to other ML (Fig. 2), it is not surprising to observe major differences between the phylogenetic tree constructed in the study and other trees using different allergens (Cui et al., 2012; Sun et al., 2016) as well as a tree using 12S rRNA (Suarez-Martinez et al., 2005). However, when comparing it to a phylogenetic tree using nuclear rDNA and other protein-coding genes (Klimov and OConnor, 2013), there are some similarities. One of which is the closeness of E. maynei to the Dermatophagoides species to each other. Another similarity is the distance of the genus Suidasia to E. maynei and the Dermatophagoides sp. Still, discrepancies were observed. This suggests that the construction of a phylogenetic tree using the HDM group 2 allergen is not recommended for phylogenetic determination. This is not surprising since the functionality of the protein is highly dependent on its structure and the protein properties of some individual amino acids in various important sites (Ohto et al., 2012).

Because the sequence is not strictly conserved, some HDM group 2 allergen might have an effect on the elicitation of the innate immune system of an individual. Der $\mathrm{p} 2$, in particular, showed functional homology to MD-2, despite having a low sequence homology. So much so that it can stimulate the TLR4-LPS-MD-2 pathway in MD-2-deficient mice while triggering allergic asthma (Trompette et al., 2009). Der f 2, like the previous allergen, was found to have the ability to bind to LPS from E. coli. Although inconclusive, Der f 2 might bind to the TLR4 like the previous allergen due to an almost identical amino acid sequence to Der $\mathrm{p} 2$ (88\%) (Ichikawa et al., 2009). Thus, in order to properly assess the clinical relevance of Sui p 2, analysis of its protein folding, not only on its epitopes, are necessary.

Based from the in silico rendering of its surface, Sui p 2 appears to have hydrophobic amino acids situated and directed towards its cavity (Fig. 3 and 4). These amino acid residues are essential for lipid-binding and LPS recognition, since it allows the binding, through hydrophobic interaction, of each acyl chain of the LPS to the hydrophobic amino acids within the cavity (Ohto et al., 2012; Oblak and Jerala, 2015). However, the ability of the hydrophobic residues of Sui p 2 to surround each acyl chain cannot be determined on the model alone. This is due to possible changes in configuration and structure when LPS binds to Sui $\mathrm{p} 2$. For example, the Der $\mathrm{f} 2$ allergen must undergo a clamshell-like motion when binding to ligand, the LPS. This will increase the overall area of its hydrophobic cavity to accommodate the said ligand (Ichikawa et al., 2005). Nevertheless, a strongly hydrophobic internal cavity present in Suip2.01 is enough to suggest that LPS can bind to Sui p 2 (Horáčková et al., 2010; Ohto et al., 2012; Ichikawa et al., 2009).

Furthermore, Tyr102 of both mMD-2 and hMD-2 is important to facilitate lipid-binding and anchoring, especially to lipid IVa, a precursor of LPS. The said amino acid binds to the 3-OH of the R3' acyl chain of Lipid IVa through hydrogen bonding (Ohto et al., 2012).

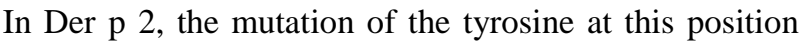
ablated its ability to elicit LPS-induced TLR4 signaling (Trompette et al., 2009). However, Der p 2 was shown to bind to LPS despite having a phenylalanine instead of a tyrosine, suggesting this is not important for the ability of the HDM group 2 allergens to mimic MD-2 (Ichikawa et al., 2009). Sui p 2 has a histidine, rather than tyrosine or phenylalanine, on the $74^{\text {th }}$ amino acid parallel to Tyr102 of MD-2. Due to the imidazole ring found in histidine, His74 could act as a recipient for hydrogen bonding to the 3-OH acyl chain of lipid Iva. Despite contrasting claims on the importance of this region, His74 can serve as an anchoring site for LPS leading it to have a greater affinity to Sui p 2 .

In order to elicit the mammalian immune system response, MD-2 binds to TLR4 through hydrogen bonding and enhanced by electrostatic potentials at the binding site of both molecules. The positively charged Lys109 and Arg68 of mammalian MD-2 binds to the negatively charged Asp83 and Asp41 at the A patch of the TLR4, respectively (Horackova et al., 2010). However, only Lys109 is conserved in the considered HDM group 2 allergens. Among the other conserved TLR4 binding sites considered (Ohto et al., 2012; Walsh et al., 2008), only Gly110 of the mammalian MD-2 is conserved in all of the considered allergens. Still, despite the low homology 
of its binding sites, TLR4 can still function due to its interaction to the MD-2-bounded LPS. Two phosphate groups of the LPS at the mouth of the MD-2 cavity, specifically 1-PO4 and 4'-PO4, make a direct hydrogen bond to Lys360 and Lys263 of the TLR4, respectively (Ohto et al., 2012). Also, it has been shown that LPSinduced inflammation can be inhibited by LPS antagonists that either compete with other LPS molecules for MD-2 pocket entry or the removal of necessary amino acids that anchor it to MD-2 (Park et al., 2012; Koo et al., 2013). Thus, it can be inferred that Sui p 2 can bind to TLR4 because of its homology to important amino acids of the said allergens as well as its deduced ability to bind to LPS.

LPS, bounded to MD-2, is also responsible for the dimerization of TLR4/MD-2/LPS complex to another TLR4'/MD-2'/LPS' complex, which is important for the initiation of the innate immune system through LPS. Two of the important amino acid residues of the mMD-2 for TLR4 and TLR4' binding is the Leu125 and Pro127. Leu125 of mMD-2 made a Van der Waals contacts to Asn415, Met417, Leu442 and Ser443 of TLR4'. Whereas Pro127 of mMD-2, Ser127 of hMD-2, made a Van der Waals interaction to Leu442 of the same TLR4'. These two amino acids are important to reinforce the hydrophobic interaction in the peripheral region of the dimerization interface of MD-2 to either TLR4 or TLR4' (Ohto et al., 2012). Pro127 and Leu127 can be seen in all of the HDM group 2 allergens, suggesting its capability to bind to another TLR4/MD-2/LPS complex for dimerization. Other considered amino acids are either conserved (Val82 and Leu87 of the mammalian MD-2) or semi-conserved in the HDM group 2 allergens. It was suggested that the conservation of the remaining amino acids is due to the properties of its sidechains (Walsh et al., 2008).

Hence, even if the TLR4 and TLR4' binding sites on Sui p 2 is incomplete, it may still bind to TLR4 and TLR4' and induce the innate immune system (Fig. 6). Like Sui p 2, Der p 2 and Der f 2 has an incomplete TLR4 and TLR4' binding sites. However, these allergens were proven to bind to LPS and induce the LPS-driven TLR4 signaling in MD-2 deficient mice (Trompette et al., 2009; Ichikawa et al., 2009). Considering that TLR4 binding is needed for proper induction of the LPS-driven TLR4 signaling (Park et al., 2012; Jeong et al., 2015), it can be inferred that TLR4 can still bind to HDM group 2 allergen in spite of a relatively incomplete TLR4 binding sites. Additionally, TLR4/MD-2 dimerization complex does not depend entirely on the TLR4 and TLR4' binding sites. MD-2-bounded LPS also has a role in the said dimerization through electrostatic, hydrogen and or hydrophobic interaction (Ohto et al., 2012; Jeong et al., 2015). This can compensate the loss of some of the TLR4 and TLR4' binding sites in HDM group 2 allergen and ultimately, in Sui p 2.
As an allergen, Sui p 2 has conserved amino acids that were proven to be epitopes of Der $\mathrm{f} 2$ and Der $\mathrm{p} 2$. Based on the sequence comparison of Sui $\mathrm{p} 2$ to both Der f 2 and Der p 2, it can be deduced that the Sui $\mathrm{p} 2$ epitopes conformational in nature due to its highly distributed putative epitope-forming amino acids (Fig. 7). These findings are expected considering that the epitopes of both Der p 2 and Der f 2 are both conformational (Szalai et al., 2007; Ichikawa et al., 2009). Additionally, cross-reactivity of Sui p 2 to either Der f 2 and Der p 2 can be assumed due to the conservation of some of the amino acids that are important for epitope formation. It is also expected that binding activity of Sui $\mathrm{p} 2$ is the highest among the other allergens from $\mathrm{Sp}$ considering that the HDM group 2 allergen exhibits the greatest IgE binding affinity when compared to all other HDM allergens (Thomas, 2015; Jeong et al., 2012; Hong et al., 1994). However, the true IgE binding activity of Sui p 2 cannot be determined based on the sequence comparison alone due to possible epitope-forming amino acids that are unique to Sui $\mathrm{p} 2$.

Furthermore, it was deduced that Sui p 2 is a thermostable protein. Hence, these deduced conformational epitopes will be preserved even if it is exposed in the harsh environment outside the source organism (Vanga et al., 2018; Huschek et al., 2016; Pfeifer et al., 2015). Also, it was reported that subjecting a thermostable allergen to heat treatment can increase its IgE binding affinity due to the slight change in its conformation, which grants better accessibility to otherwise hidden epitopes (Pfeifer et al., 2015). This, together with the fact that Sp allergens can be seen in stored food (Mariana et al., 2000; Sanchez-Borges et al., 2013), increases the chance of sensitizing atopic individuals and eliciting both their allergic and innate immune responses.

\section{Conclusion}

The ML lipid-binding domain-homolog cDNA gene from Suidasia pontifica is a $550 \mathrm{bp}$ gene that contains a 405 bp Open Reading Frame with 3' and 5' untranslated regions. Sui p 2 gene sequence showed sequence homology to known HDM group 2 allergen genes and to mammalian MD-2, respectively. The Sui p 2 gene encodes 134 amino acid long proteins with a signal peptide attached to it. Tertiary (3D) structure model prediction of Sui $\mathrm{p} 2$ revealed the distinctive immunoglobulin fold of ML lipid-binding domain family. Sui p 2 contains two $\beta$-sheets consisting of three and four $\beta$-strands per sheet. Sui p 2 also contains one $\alpha$ helix and two nonparallel $\beta$-strands. The immunoglobulin fold was stabilized by three pairs of disulfide bridge-forming cysteine residues. A putative hydrophobic cavity at the center of the protein has been noted, thus, lipid-binding can be inferred. Sui $\mathrm{p} 2$, despite having a relatively low sequence homology to 
other allergens, retained the structural conformation homologous to the ML protein family. The majority of the TLR4 and TLR4' binding sites are conserved in Sui p 2, similar to other HDM allergens that were proven to bind to TLR4 and LPS. Thus, functional homology of Sui $\mathrm{p} 2$ to MD-2 can be deduced. As a possible HDM group 2 allergen, some of the amino acids in Sui $\mathrm{p} 2$ has a potential to form epitopes that are conformational in nature. Overall, 20 epitope-forming amino acids were identified in Sui p 2, 16 of which are accessible for $\operatorname{IgE}$ binding. Hence, Sui p 2 has potential to be one of the major allergens of Suidasia pontifica.

The results presented herein suggest that Sui p 2 may play an important role in HDM induced allergy. Considering that it is one of the serodominant allergens of HDMs and its capability to induce the mammalian innate immune system, its elucidation will greatly increase our understanding about Suidasia pontifica and its clinical importance. This can also pave the way to the expression of the recombinant Sui p 2 allergen, which can be used for allergy component resolved diagnosis and allergen-specific immunotherapy.

\section{Acknowledgment}

The authors would like to extend their greatest appreciation and sincerest thanks to the people and institutions that provided him the necessary help and support needed to complete the study: M. Maningas, M. Sabit, J. Yap, L. Medina, R. Velasco and all of the people in the molecular biology lab in UST-TARC.

\section{Funding Information}

This study was funded and supported by the Department of Science and Technology and the University of Santo Tomas - Thomas Aquinas Research Complex. The funding source was not involved in the design, collection of data, analysis and interpretation of the said data, the decision to submit the study for publication, etc.

\section{Author's Contribution}

Joshua Evans M. Bajao: Performed the experiments, designed the study, collected all the data, analyzed and interpreted the data and wrote the manuscript.

John Donnie A. Ramos: Verified all the data, provided technical support, funded the project and edited the manuscript.

\section{Ethics}

This article is original and contains unpublished material. The corresponding author confirms that all of the other authors have read and approved the manuscript and no ethical issues involved.

\section{Reference}

Altschul, S.F., W. Gish, W. Miller, E.W. Myers and D.J. Lipman, 1990. Basic local alignment search tool. J. Mol. Biol., 215: 403-410. DOI: $10.1016 / \mathrm{S} 0022-2836(05) 80360-2$

Arnold, K., L. Bordoli, J. Kopp and T. Schwede, 2006. The SWISS-MODEL workspace: A web-based environment for protein structure homology modelling. Bioinformatics, 22: 195-201. DOI: $10.1093 /$ bioinformatics/bti770

Benkert, P., S.C.E. Tosatto and D. Schomburg, 2008. QMEAN: A comprehensive scoring function for model quality assessment. Proteins: Structure Funct. Bioinform., 71: 261-277. DOI: 10.1002/prot.21715

Bergmann, K., J. Heinrich and H. Niemann, 2016. Current status of allergy prevalence in Germany. Allergo J. Int., 25: 6-10. DOI: 10.1007/s40629-016-0092-6

Bork, P., L. Holm and C. Sander, 1994. The immunoglobulin fold: Structural classification, sequence patterns and common core. J. Mol. Biol., 242: 309-320. DOI: 10.1006/jmbi.1994.1582

Ciftci, I.H., Z. Cetinkaya, M. Atambay, N. Kiyildi and O.M. Aycan et al., 2006. House dust mite fauna in western Anatolia, Turkey. Korean J. Parasitol., 44: 259-264. DOI: 10.3347/kjp.2006.44.3.259

Cui, Y., Y. Zhou, G. Ma, L. Yang and Y. Wang et al., 2012. Cloning, bioinformatics analysis and expression of the dust mite allergen of Der $f 5$ of Dermatophagoides farinae. Braz. J. Med. Biol. Res., 45: 746-752.

DOI: 10.1590/S0100-879X2012007500077

Cui, Y., Y. Zhou, W. Shi, G. Ma and L. Yang et al., 2010. Cloning, expression and analysis of the group 2 allergen from Dermatophagoides farina from China. An. Acad. Bras Cienc., 82: 941-51. DOI: 10.1590/s0001-37652010000400017

Dall'Antonia, F., T. Pavlov-Keller, K. Zangger and W. Keller, 2014. Structure of allergen and structure based epitope prediction. Methods, 66: 3-21. DOI: 10.1016/j.ymeth.2013.07.024

Farrokhi, S., M.K. Chebi, A. Movahed, R. Tahmasebi and D. Iranpour et al., 2015. Common aeroallergens in patients with asthma and allergic rhinitis living in southwestern part of Iran: Based on skin prick test reactivity. Iran J. Allergy Asthma Immunol., 14: 133-138.

Gasteiger, E., A. Gattiker, C. Hoogland, I. Ivanyi and R.D. Appel et al., 2003. ExPASy: The proteomics server for in-depth protein knowledge and analysis. Nucleic Acids Res., 31: 3784-3788. DOI: $10.1093 /$ nar/gkg563

Gasteiger, E., C. Hoogland, A. Gattiker, S. Duvaud and M.R. Wilkins et al., 2005. Protein Identification and Analysis Tools on the ExPASy Server. In: The Proteomics Protocols Handbook, Walker, J.M. (Ed.), Humana Press, pp: 571-607. 
Hanski, I., L. von Hertzen, N. Fyhrquist, K. Koskinen and K. Torppa et al., 2012. Environmental biodiversity, human microbiota and allergy are interrelated. PNAS, 109: 8334-8339. DOI: 10.1073/pnas.1205624109

Hernández-Cadena, L., D.C. Zeldin, A. BarrazaVillarreal, M.L. Sever and P.D. Sly et al., 2015. Indoor determinants of dustborne allergens in Mexican homes. Allergy Asthma Proc., 36: 130-137. DOI: 10.2500/aap.2015.36.3801

Hong, C., J.W. Park and D.H. Nahm, 1994. Measurement of $\operatorname{IgE}$ and $\operatorname{IgG}$ subclass antibodies to whole body antigen and two major allergens (Der fI and Der fII) of Dermatophagoides farinae in normal subjects and asthmatics. Yonsei Med. J., 35: 453-463. DOI: $10.3349 / y m j .1994 .35 .4 .453$

Horáčková, J., N. Rudenko, M. Golovchenko and L. Grubhoffer, 2010. Der-p2 (Dermatophagoides pteronyssinus) allergen-like protein from the hard tick Ixodes Ricinus-a novel member pf ML (MD-2-related lipid-recognition) domain protein family. Parasitology, 137: 1139-11349. DOI: 10.1017/S0031182009992083

Horackova, J., N. Rudenko, R.M. Golovchenko and L. Grubhoffer, 2010. Der-p2 (Dermatophagoides pteronyssinus) allergen-like protein from the hard tick Ixodes ricinus - a novel member of ML (MD-2-related lipid-recognition) domain protein family. Parasitology, 137: 1139-1149. DOI: 10.1017/S0031182009992083

Huschek, G., J. Bönick, Y Löwenstein, S. Sievers and H. Rawel, 2016. Quantification of allergenic plant traces in baked products by targeted proteomics using isotope marked peptides. Food Sci. Technol., 74: 286-293. DOI: 10.1016/j.lwt.2016.07.057

Ichikawa, S., T. Takai, T. Inoue, T. Yuuki and Y. Okumura et al., 2005. NMR study on the major mite allergen Der f 2: Its refined tertiary structure, epitopes for monoclonal antibodies and characteristics shared by ML protein group members. J. Biochem., 137: 255-263. DOI: 10.1093/jb/mvi039

Ichikawa, S., T. Takai, T. Yashiki, S. Takahashi and K. Okumura et al., 2009. Lipopolysaccharide binding of the mite allergen Der f 2. Genes Cells, 14: 1055-1065. DOI: $10.1111 /$ j.1365-2443.2009.01334.x

Jeong, K.Y., J.P.M. Park and C. Hong, 2012. House dust mite allergy in Korea: The most important inhalant allergen in current and future. Allergy Asthma Immunol. Res., 4: 313-325.

DOI: 10.4168/aair.2012.4.6.313

Jeong, K.Y., J.Y. Lee, M. Son, M. Yi and T. Yong et al., 2015. Profiles of IgE sensitization Der f 1, Der f 2, Der f 6, Der f 8, Der f 10 and Der f 20 in Korean house dust mite allergy patients. Allergy Asthma Immunol. Res., 7: 483-488. DOI: 10.4168/aair.2015.7.5.483

Jeong, K.Y., J.Y. Lee, M. Son, M. Yi and T. Yong et al., 2015. Profiles of IgE sensitization Der f 1, Der f 2, Der f 6, Der f 8, Der f 10 and Der f 20 in Korean house dust mite allergy patients. Allergy Asthma Immunol. Res., 7: 483-488. DOI: 10.4168/aair.2015.7.5.483
Källberg, M., H. Wang, S. Wang, J. Peng and Z. Wang et al., 2012. Template-based protein structure modeling using the RaptorX web server. Nature Protocols, 7: 1511-1522. DOI: 10.1038/nprot.2012.085

Kelley, L.A., S. Mezulis, C.M. Yates, M.N. Wass and M.J.E. Sternberg, 2015. The Phyre2 web portal for protein modeling, prediction and analysis. Nature Protocols, 10: 845-858. DOI: 10.1038/nprot.2015.053

Klimov, P.B. and B. OConnor, 2013. Is permanent parasitism reversible? - critical evidence from early evolution house dust mites. Syst. Biol., 62: 411-423. DOI: $10.1093 /$ sysbio/syt008

Koo, J.E., Z. Park, N.D. Kim and J.Y. Lee, 2013. Sulforaphane inhibits the engagement of LPS with TLR4/MD2 complex by preferential binding to Cys133 in MD2. Biochem. Biophys. Res. Commun., 434: 600-605. DOI: 10.1016/j.bbrc.2013.03.123

Kumar, S., G. Stetcher and K. Tamura, 2016. MEGA7: Molecular evolutionary genetics analysis version 7.0 for bigger datasets. Molecular Biol. Evolut., 33: 1870-1874.

Lau, M.Y.Z., S.C. Dharmage, J.A. Burgess, A.J. Lowe and C.J. Lodge et al., 2014. CD14 polymorphisms, microbial exposure and allergic diseases: A systematic review of gen-environment interactions. Allergy, 69: 1440-1453. DOI: 10.1111/all.12454

Mariana, A., S.K. Heah, A.L. Wong and T.M. Ho, 2010. The occurrence of arthropods in processed rice products in Malaysia. Asian Pacific J. Tropical Med., 2010: 552-554. DOI: 10.1016/S1995-7645(10)60133-2

Mariana, A., T.M. Ho, B.S. Gendeh, H. Iskandar and M. Zainuldin-Taib, 2000. First report on sensitization to allergens of a house dust mite, Suidasia pontifica (Acari: Saproglyphidae). Southeast Asian J. Trop. Med. Public Health, 31: 722-733.

Nielsen, M., C. Lundegaard, O. Lundo and T.N. Petersen, 2010. CPHmodels-3.0-remote homology modeling using structure guided sequence profiles. Nucleic Acids Res., 38: W576-W581. DOI: $10.1093 /$ nar/gkq535

Oblak, A. and R. Jerala, 2015. The molecular mechanism of species-specific recognition of lipopolysaccharides by the MD-2/TLR4 receptor complex. Molecular Immunol., 63: 134-142. DOI: 10.1016/j.molimm.2014.06.034

Ohto, U., K. Fukase, K. Miyake and T. Shimizu, 2012. Structural basis of species-specific endotoxin sensing by innate immune receptor TLR4/MD-2. PNAS, 109: 7421-7426. DOI: 10.1073/pnas. 1201193109

Park, S.H., N.D. Kim, J. Jung, C. Lee and S. Han et al., 2012. Myeloid differentiation 2 as a therapeutic target of inflammatory disorders. Pharmacol. Therapeut., 133: 291-298. DOI: 10.1016/j.pharmthera.2011.11.001

Pawankar, R., C. Bunnag, N. Khaltaev and J. Bousquet, 2012. Allergic rhinitis and its impact on asthma in Asia pacific and the ARIA update 2008. WAO J., 5: S212-S217. DOI: 10.1097/WOX.0b013e318201d831 
Peters, R.L., K.J. Allen, S.C. Dharmage, M.L.K. Tang and J.J. Koplin et al., 2013. Skin prick test responses and allergen-specific IgE levels as predictors of peanut, egg and sesame allergy in infants. J. Allergy Clin. Immunol., 132: 874-80. DOI: 10.1016/j.jaci.2013.05.038

Petersen, T.N., S. Brunak, G. Von Heijne and H. Nielsen, 2011. Discriminating signal peptides from transmembrane regions. Nature Meth., 8: 785-786. DOI: $10.1093 / \mathrm{molbev} / \mathrm{msw} 054$

Pettersen, E.F., T.D. Goddard, C.C. Huang, G.S. Couch and D.M. Greenblatt et al., 2004. UCSF Chimera - a visualization system for exploratory research and analysis. J. Comput. Chem., 25: 1605-1612. DOI: $10.1002 /$ jcc.20084

Pfeifer, S., M. Bublin, P. Dubiela, K. Hummel and J Wortmann et al., 2015. Cor a 14, the allergenic $2 \mathrm{~S}$ albumin from hazelnut, is highly thermostable and resistant to gastrointestinal digestion. Mol. Nutr. Food Res., 59: 2077-2086. DOI: 10.1002/mnfr.201500071

Pontius, J., J. Richelle and S.J. Wodak, 1996. Deviations from standard atomic volumes as a quality measure for protein crystal structures. J. Mol. Biol., 264: 121-136. DOI: 10.1006/jmbi.1996.0628

Ramos, J.D.A., M.P.S. Castillo, M. del Rosario, M.A.S. Gapay and T.P. Go et al., 2007. Allergenicity and cross reactivity of 3 house dust mite species among allergic patients. Philippine J. Sci., 136: 139-146.

Robert, X. and P. Gouet, 2014. Deciphering key features in protein structures with the new ENDscript server. Nucl. Acids Res., 42: W320-W324. DOI: $10.1093 /$ nar/gku316

Ryu, J., S.J. Kim, S. Rah, J.I. Kang and H.E. Jung et al., 2017. Reconstruction of LPS transfer cascade reveals structural determinants within LBP, CD14 and TLR4-MD2 for efficient LPS recognition and transfer. Immunity, 46: 38-50.

DOI: 10.1016/j.immuni.2016.11.007

Sanchez-Borges, M., R. Suarez-Chacon, A. CaprilesHulett, F. Caballero-Fonseca and E. FernandezCaldez, 2013. Anaphylaxis from ingestion of mites: pancake anaphylaxis. J. Allergy Clin. Immunol., 131: 31-35. DOI: 10.1016/j.jaci.2012.09.026

Sievers, F., A. Wilm, D. Dineen, T.J. Gibson and K. Karplus et al., 2011. Fast, scalable generation of high-quality protein multiple sequence alignments using Clustal Omega. Molecular Syst. Biol., 7: 539-539. DOI: 10.1038/msb.2011.75

Suarez-Martinez, E.B., F. Montealegre, J.M. SierraMonte and R.J. Herrera, 2005. Molecular identification of pathogenic house dust mites using 12S rRNA sequences. Electrophoresis, 26: 2927-2934. DOI: 10.1002/elps.200410353

Sun, X., Z. Li, G. Dong, W. Yin and S. Li et al., 2016. Molecular characterization of the group 9 allergen of Dermatophagoides farinae. Int. J. Clin. Exp. Med., 9: 17175-17186.
Szalai, K., J. Fuhrmann, T. Pavkov, M. Scheidl and J. Wallmann et al., 2007. Mimotopes identify conformational B-cell epitopes on the two major house dust mite allergens Der p 1 and Der p 2 . Molecular Immunol., 45: 1308-1317.

DOI: 10.1016/j.molimm.2007.09.012

Thomas, W.R., 2015. Hierarchy and molecular properties of house dust mite allergens. Allergology Int., 64: 304-311. DOI: 10.1016/j.alit.2015.05.004

Trompette, A., S. Divanovic, A. Visintin, C. Blanchard and R.S. Hedge et al., 2009. Allergenicity resulting from functional mimicry of a toll-like receptor complex protein. Nature, 457: 585-588.

DOI: $10.1038 /$ nature 07548

Vanga, S.K., M. Jain and V. Raghavan, 2018. Significance of fruit and vegetable allergens: Possibilities of its reduction through processing. Food Rev. Int., 34: 103-125. DOI: 10.1080/87559129.2016.1239208

Wallner, B. and A. Elofsson, 2003. Can correct protein models be identified? Protein Sci., 12: 1073-1086. DOI: $10.1110 /$ ps.0236803

Walsh, C., M. Gangloff, T. Monie, T. Smyth and B. Wei et al., 2008. Elucidation of the MD-2/TLR4 interface required for signaling by lipid IVa. J. Immunol., 181: 1245-1254. DOI: 10.4049/jimmunol.181.2.1245

Wang, M., Y. Zhang, D. Han and L. Zhang, 2012. Association between polymorphisms in cytokine genes IL-17A and IL-17F and development of allergic rhinitis and comorbid asthma in Chinese subjects. Human Immunol., 73: 647-653.

DOI: 10.1016/j.humimm.2012.03.010

Wiederstein, M. and M.J. Sippl, 2007. ProSA-web: Interactive web service for the recognition of errors in three-dimensional structures of proteins. Nucleic Acids Res., 35: W407-W410. DOI: 10.1093/nar/gkm290

Yap, J.M.G., M.W. Ching, R.S. Cruz and J.D.A. Ramos, 2014. Specific IgE against the house dust mite Suidasia pontifica as a risk factor for asthma and allergies in the tropics. Acta Manilana, 62: 1-8.

Yong, S.B., C.C. Wu, Y.C. Tzeng, W.C. Hung and K.D. Yang, 2013. Different profiles of allergen sensitization in different ages and geographic areas in Changhua, Taiwan. J. Microbiol. Immunol. Infect., 46: 295-301. DOI: 10.1016/j.jmii.2012.07.002 